\title{
CAPACITÉS D'ACTION DES AGRICULTEURS DANS LES PROJETS LOCAUX DE DÉVELOPPEMENT AGRICOLE AU MAROC
}

Nicolas Faysse, Sara El Mkadmi, Mostafa Errahj

\author{
Armand Colin | « Revue Tiers Monde»
}

2015/4 N²24 | pages 143 à 162

ISSN 1293-8882

ISBN 9782200930165

Article disponible en ligne à l'adresse :

http://www.cairn.info/revue-tiers-monde-2015-4-page-143.htm

\section{!Pour citer cet article :}

Nicolas Faysse et al., «Capacités d'action des agriculteurs dans les projets locaux de développement agricole au Maroc », Revue Tiers Monde 2015/4 (N²24), p. 143-162.

Distribution électronique Cairn.info pour Armand Colin.

(C) Armand Colin. Tous droits réservés pour tous pays.

La reproduction ou représentation de cet article, notamment par photocopie, n'est autorisée que dans les limites des conditions générales d'utilisation du site ou, le cas échéant, des conditions générales de la licence souscrite par votre établissement. Toute autre reproduction ou représentation, en tout ou partie, sous quelque forme et de quelque manière que ce soit, est interdite sauf accord préalable et écrit de l'éditeur, en dehors des cas prévus par la législation en vigueur en France. Il est précisé que son stockage dans une base de données est également interdit. 
VARIA

\title{
CAPACITÉS D'ACTION DES AGRICULTEURS DANS LES PROJETS LOCAUX DE DÉVELOPPEMENT AGRICOLE AU MAROC
}

\author{
Nicolas Faysse $^{\star}$, Sara El Mkadmi ${ }^{\star *}$, Mostafa Errahj $^{\star \star \star}$
}

Au Maroc, de nouvelles politiques publiques prévoient une conception participative des projets de développement agricole et rural au niveau local. L'étude porte sur la participation des agriculteurs de deux villages à la conception et la mise en œuvre d'un projet oléicole, et sur l'amélioration de leurs capacités d'action que cette participation a permise. Les agriculteurs n'ont eu qu'une influence faible sur la conception et la mise en œuvre du projet. Dans un des deux villages, les agriculteurs ont cependant appris comment obtenir des financements publics et ont par la suite mené à bien plusieurs projets. Les nouvelles politiques publiques peuvent ainsi induire une forte différenciation entre collectifs d'agriculteurs, notamment dans leur capacité à prendre des initiatives vis-à-vis de l'administration.

Mots clés : Agencéité, Maroc, organisation professionnelle agricole, plan Maroc vert, projet de développement.

\section{INTRODUCTION}

Le Maroc a connu ces dernières années un renouveau des politiques publiques pour le développement rural et agricole. Il s'agit pour l'essentiel de l'Initiative nationale de développement humain (INDH) depuis 2005 (Bergh, 2012 ; De Miras, 2007) et du plan Maroc vert depuis 2008 (Faysse, 2015). Ces politiques agissent pour l'essentiel par le biais de projets de développement au niveau local et affichent l'ambition de concevoir et de mettre en ouvre ces projets

\footnotetext{
* Socioéconomiste, chercheur au Cirad, UMR G-Eau, faysse@cirad.fr.

** Ingénieur agronome, Crédit agricole du Maroc.

*** Socioéconomiste, enseignant-chercheur à l'École nationale d'agriculture de Meknès, Maroc.
} 
en coordination avec une entité représentant les bénéficiaires. Au cours des années 1990, des démarches qui se voulaient participatives avaient déjà été utilisées dans plusieurs projets de développement au niveau local, financés par des bailleurs de fonds internationaux (par exemple : Kadiri, 2012). L'INDH et le plan Maroc vert innovent dans la mesure où ces politiques ambitionnent d'institutionnaliser ces démarches participatives, dans le cadre de procédures formalisées et pérennes pour la conception et la mise en œuvre de projets.

Cette évolution offre, a priori, des opportunités pour que les habitants des zones rurales jouent un rôle actif dans la conception et la mise en œuvre des projets de développement, en particulier parce que l'utilisation de procédures formalisées et pérennes pourrait permettre aux habitants des zones rurales de mieux comprendre le fonctionnement de ces pratiques et, par là, de mieux gérer leur relation avec les institutions publiques de développement.

Au Maroc, les études sur les projets de développement qui annonçaient une approche participative ont surtout cherché à qualifier si ces projets organisaient, en pratique, la participation des bénéficiaires au niveau de ce qui était initialement annoncé (par exemple: El Jihad, 2010). Cependant, d'une part, de telles études tendent à centrer leur analyse sur le point de vue des institutions de développement et, d'autre part, ces études n’ont mesuré en général que l'influence ponctuelle qu'avaient pu avoir les bénéficiaires sur le projet de développement. Pour analyser des dispositifs de développement agricole et rural qui s'annoncent comme participatifs, il est intéressant de mener une analyse centrée sur le point de vue des bénéficiaires et de qualifier la capacité de ces derniers à gérer les relations avec les autres acteurs dans de tels dispositifs. Cette capacité s'est constituée avant le projet et évolue ensuite au cours et à l'issue du projet. Cette capacité des bénéficiaires peut être conceptualisée comme faisant partie de leur agencéité, c'est-à-dire la capacité des individus de disposer de marges de manœuvre et d'agir, dans le sens qu'ils pourraient « à n'importe quel moment d'une séquence donnée, avoir agi différemment » (Giddens, 1984).

Certaines études sur les projets de développement au Maroc se sont focalisées sur les bénéficiaires de ces projets, mais ces études ont avant tout porté sur des projets ponctuels (par exemple, Kadiri, 2012 ; Montanari et Bergh, 2014) et non sur des dispositifs pérennes de conception et de mise en œuvre de projets de développement qui s'affichent comme participatifs.

Cet article a pour objectif d'étudier quelle est l'agencéité des agriculteurs dans les projets conçus dans le cadre des nouvelles politiques publiques de développement rural et agricole au Maroc. L'étude a porté sur un projet d'intensification et de valorisation de la production d'olives dans deux villages du Nord du Maroc. Nous avons cherché à comprendre dans quelle mesure les agriculteurs ont pu obtenir ce qu'ils désiraient durant la conception et la mise en œuvre du projet, et comment ils ont appris de ce projet pour pouvoir 
éventuellement concevoir d'autres actions de développement et obtenir des financements pour ces projets dans le cadre des nouvelles politiques publiques.

\section{POLITIQUES PUBLIQUES ET AGENCÉITÉ DES AGRICULTEURS AU MAROC}

Nous présentons ici brièvement la façon dont les principales politiques publiques de développement rural et agricole au Maroc, passées et actuelles, ont envisagé la participation des agriculteurs, et les études qui ont été menées sur l'agencéité des agriculteurs dans le cadre de ces politiques publiques. Après l'indépendance, l'État marocain a conçu la grande majorité de ses actions de développement rural et agricole avec une participation très limitée des agriculteurs. Cette faible implication des agriculteurs peut être expliquée par l'étendue large de ces projets, tels que le projet Sebou (Lazarev, 2012). Mais surtout, cette faible implication a correspondu à un objectif d'affirmation de la présence de l'État et de contrôle politique (Leveau, 1976). L'administration a conçu quelques projets à un niveau local à partir des années 1990, mais toujours avec une faible participation des agriculteurs (Direction des aménagements forestiers, 2002).

Dans un tel contexte, les agriculteurs marocains ont parfois utilisé des stratégies d'évitement ou de résistance lors de la mise en œuvre des projets (Préfol, 1986). Dans les périmètres de grande hydraulique, où l'influence de l'État a été par le passé très forte, les agriculteurs ont développé des capacités d'influence par le biais de contacts informels avec des membres de l'administration (Bouzidi, 2012). Ces actions sont cependant menées dans un cadre informel. Dans les périmètres de grande hydraulique, l'État continue de considérer les agriculteurs comme des bénéficiaires et non des partenaires (Faysse et al., 2010).

Plus récemment, différentes actions publiques pour le développement rural au Maroc ont conçu leur intervention sur la base de projets de développement au niveau local et ont, plus ou moins selon les cas, formalisé une participation des agriculteurs à la conception et à la mise en œuvre de ces projets. Ces actions se fondent sur un "populisme bureaucratique» (Chauveau, 1994), c'est-à-dire la volonté d'associer une action publique rationnelle et efficace avec une participation de la communauté des bénéficiaires. Ces actions peuvent être regroupées en trois ensembles, selon qu'elles sont financées par des bailleurs de fonds internationaux, par l'INDH ou par le plan Maroc vert.

Premièrement, plusieurs projets de développement ont été financés dans le cadre d'une coopération entre l'administration marocaine et des bailleurs de fonds internationaux, ces derniers conditionnant leur appui financier à la mise en œuvre d'une démarche participative. Différentes études ont été menées sur ces projets et ont abouti à des résultats très différents. D'un côté, El Jihad (2010) et Montanari et Bergh (2014) décrivent des situations de très faibles implications 
des bénéficiaires dans la conception des projets. De l'autre, Kadiri (2012) montre comment, en une dizaine d'années, des associations d'usagers d'eau agricole ont évolué d'une situation de passivité par rapport à l'administration à une situation où ces associations jouent un rôle central pour le développement agricole de leur région.

Deuxièmement, un des axes de financement de l'INDH est un appel à projets pour des activités génératrices de revenus. Chaque année, des associations et des coopératives peuvent élaborer des propositions de projets qui sont ensuite sélectionnées, selon une procédure formalisée, au niveau de comités provinciaux où siègent des représentants des associations et coopératives de chaque province (Bergh, 2012). Bergh (2012) et Bono (2012) font le même constat, à savoir qu'il n'y a pas de co-construction des projets de développement entre agriculteurs et administration de l'INDH, mais que la relation entre ces acteurs reste fondée sur une logique de distribution de ressources matérielles. Dans cette logique de "guichet ", les associations ou coopératives bénéficiaires évitent d'être en situation de conflit avecl'administration, pour ne pas mettre en péril la possibilité d'obtenir des financements dans le futur.

Troisièmement, le plan Maroc vert constitue, comme l'INDH, une politique structurante sur le long terme car il doit durer au moins jusqu'à 2020. Ce plan définit deux agricultures, une à vocation productiviste (qualifiée de Pilier I) et une autre qualifiée de "solidaire» ou de Pilier II (Faysse, 2015). Le plan Maroc vert a progressivement mis en place une procédure pour la conception et la mise en œuvre des projets du Pilier II (Agence de développement agricole, 2015). Thomas (2013) a étudié la conception de deux de ces projets du Pilier II et montre que, dans les cas étudiés, les agriculteurs ont fait preuve de fortes capacités pour en négocier le contenu. En particulier, lorsque les agriculteurs ont été en désaccord avec les propositions de l'administration lors de la conception des projets, ils ont élaboré des contre-propositions qui intégraient les objectifs et les contraintes de l'administration. Dans un de ces deux cas, le collectif d'agriculteurs était animé par un groupe de jeunes diplômés qui n'avaient auparavant aucun lien avec l'administration.

À la différence des politiques précédentes, l'INDH et le plan Maroc vert ont ainsi défini des procédures formalisées de conception des projets de développement au niveau local et notamment de décision d'attribution des financements. La maîtrise par les agriculteurs de ces nouvelles procédures peut devenir une ressource importante pour améliorer leur agencéité et obtenir des financements pour réaliser leurs projets. 


\section{CADRE CONCEPTUEL}

L'agencéité d'un acteur s'exprime au sein d'une structure qui constitue à la fois une source de contraintes et d'opportunités (Giddens, 1984 ; Long, 2002). Plus précisément, Alsop et al. (2006) se réferent à la structure d'opportunité, définie comme les « aspects du contexte institutionnel dans lequel les acteurs opèrent qui influencent leur capacité de transformer leur agencéité en action ». Cette agencéité concerne en particulier la capacité des acteurs d'influencer les autres acteurs, notamment pour les enrôler dans leurs projets (Long, 2002), et de « s'exprimer et d'exercer une voix » (de Herdt et Bastiaensen, 2009).

Les agriculteurs peuvent exercer leur agencéité à travers des stratégies extrêmement diverses, par exemple dans le choix de leurs réponses aux questions posées par les personnes en charge des diagnostics participatifs (Mosse, 2005), ou dans une stratégie de contournement de ces dispositifs participatifs (Le Meur, 2000). Ces stratégies peuvent être fondées sur la demande de reconnaissance de droits, mais aussi sur la mobilisation de relations de clientélisme (Robins et al., 2008).

Cette agencéité peut dépendre de nombreuses ressources. Mansuri et Rao (2012) ont fait un bilan de dispositifs participatifs financés par la Banque mondiale et ont montré que, souvent, ces dispositifs bénéficient avant tout aux habitants des zones rurales qui, comparativement aux autres, ont un meilleur niveau d'études, sont plus riches et ont statut social plus élevé. Cette agencéité doit être aussi analysée de façon dynamique. Ainsi, si les projets de développement qui s'affichent comme participatifs n'aboutissent pas toujours à un renforcement des pouvoirs formels de décision des agriculteurs, ils peuvent au moins permettre aux agriculteurs de comprendre les rouages de l'administration (Corbridge et al., 2005).

\section{MÉTHODOLOGIE}

Nous avons mené des entretiens auprès de l'ensemble des acteurs intervenant dans le projet de développement étudié. Nous avons cependant opté pour un «populisme méthodologique» (Olivier de Sardan, 2008), en accordant une importance particulière au rôle des agriculteurs dans le projet. Les entretiens ont été réalisés entre juin 2011 et décembre 2012, c'est-à-dire entre un et deux ans après la date de plantation des oliviers (le projet ayant été mis en œuvre en différentes tranches). Ces entretiens ont été réalisés avec les responsables des organisations professionnelles impliquées dans le projet pour chaque village, c'est-à-dire les personnes qui ont pris part à la coordination pour la conception et la mise en œuvre du projet, à la fois avec les bailleurs de fonds et avec les 
bénéficiaires. Ces responsables ont été interviewés au moins quatre fois tout au long de l'étude.

L'enquête a aussi été menée auprès d'agriculteurs des villages concernés, de la Direction provinciale de l'agriculture (DPA) et du Centre des travaux ${ }^{1}$ de la zone d'étude et de l'un des entrepreneurs qui est intervenu dans la mise en œuvre du projet. Avec chaque acteur, l'entretien a porté sur l'historique du projet, ses objectifs et les stratégies qu'il a cherché à appliquer lors de la conception et la mise en œuvre, ses actions, et l'évaluation qu'il fait des réussites et des échecs du projet. Les agriculteurs ont aussi exprimé ce qu'ils avaient appris à l'occasion du projet, et comment ils ont pu éventuellement mobiliser ces apprentissages pour en concevoir d'autres.

\section{CAS D'ÉTUDE}

Les deux villages étudiés se trouvent à moins de $20 \mathrm{~km}$ l'un de l'autre dans une zone de petite montagne de la région du Saiss au Maroc (située entre 400 et $600 \mathrm{~m}$ d'altitude). La pluviométrie annuelle moyenne est de $580 \mathrm{~mm}$, ce qui permet de pratiquer une activité oléicole sans irrigation. Le premier village, appelé ici Arbaoua ${ }^{2}$, compte environ 2300 habitants. Les agriculteurs produisent de l'olive, des caroubes, du blé et conduisent un élevage ovin et bovin. Une enquête préliminaire (El Mkadmi, 2011) auprès de seize agriculteurs du village montre une superficie médiane cultivée de 7,7 ha par exploitation. Tous ces agriculteurs sauf un cultivaient des oliviers avant le projet (nombre médian de 130 oliviers par agriculteur). La première association du village est créée en 1987 et organise des dons de livres pour une bibliothèque locale, du soutien scolaire et de la distribution de vêtements. À partir de 2002, elle débute des activités d'alphabétisation, dans le cadre d'une convention avec le ministère de l'Éducation. L'association compte jusqu'à 350 adhérents. En 2001, une coopérative est créée, à la suggestion du président de l'association, avec vingt-quatre adhérents. En 2005, la coopérative achète une batteuse qui continue, jusqu'en 2012, d'être utilisée en commun. En 2006, la coopérative organise un achat collectif de son de blé et de feuilles de betterave pour l'alimentation du bétail de ses adhérents. La mauvaise qualité des produits achetés et le défaut de paiement de certains membres entraînent des conflits et, deux ans plus tard, dix adhérents quittent la coopérative. Les membres clés de la coopérative ont retenu de cette expérience que, pour qu'une action collective réussisse, le nombre de personnes impliquées doit être restreint. Depuis sa création, la coopérative d'Arbaoua est gérée au quotidien par trois personnes, qui ont toutes

1. Antenne locale du ministère de l'Agriculture et de la Pêche maritime et qui se nomme depuis 2013 Centre de conseil agricole.

2. Les noms des villages ont été modifiés. 
le baccalauréat (les autres membres du conseil d'administration ne jouant en pratique aucun rôle).

Le deuxième village, appelé ici Souk El-Hed, compte environ 800 habitants. Les agriculteurs du village produisent essentiellement des olives, du blé et un peu de maraîchage (le village disposant d'une source d'eau). Selon l'enquête portant sur vingt-neuf agriculteurs de ce village réalisée par El Mkadmi (2011), la superficie médiane cultivée par exploitation est de 5 ha, et vingt-sept des vingt-neuf agriculteurs interrogés avaient des oliviers avant le projet (nombre médian de 130 arbres par exploitation). En 2011, la DPA a financé le revêtement en béton des canaux traditionnels d'irrigation. Cependant, moins d'un an après la réalisation de ces travaux, des tronçons de ces canaux se sont écroulés et les agriculteurs n'avaient, en décembre 2012, pas réussi à obtenir une réparation des parties abîmées. Il n'y avait jamais eu d'association ou de coopérative dans ce village avant le projet oléicole.

\section{RÉSULTATS}

\section{Conception du projet}

Peu après le lancement du plan Maroc vert en 2008, la DPA formule un projet de renforcement de l'activité oléicole. Elle prévoit de mettre en œuvre ce projet dans trois communes rurales distinctes. L'approche se veut intégrée avec : i) la plantation de jeunes oliviers et la taille des anciens arbres ; ii) la fourniture de matériel de traitement et de récolte; iii) des formations sur la conduite de l'olivier ; iv) la fourniture d'un pressoir moderne d'olives.

La procédure d'élaboration des projets dans le cadre du plan Maroc vert stipule que la DPA contractualise avec une organisation d'agriculteurs dans chaque village, mais les bénéficiaires du projet peuvent ne pas être adhérents de cette organisation. Selon cette procédure aussi, la DPA signe des contrats avec des entrepreneurs pour la plantation et l'entretien des jeunes plants d'olivier. L'entrepreneur est en charge, pendant deux ans, de la conduite technique des jeunes plants, ce qui comprend la fertilisation, l'irrigation et le gardiennage dans la journée, pour éviter que les troupeaux de moutons ne les abîment. Les agriculteurs ne sont pas cosignataires du contrat entre la DPA et les entrepreneurs. Il n'y a pas d'espace de communication directe prévu entre les entrepreneurs et les associations, et les entrepreneurs n'ont officiellement pas à rendre de compte à ces dernières. Si elles se plaignent de la qualité du travail effectué par un entrepreneur, elles doivent en référer à la DPA.

Dans chaque commune rurale potentiellement concernée par le projet oléicole, la DPA a organisé une réunion d'information sur ce projet. Le projet 
est déjà défini lorsqu'il est présenté aux agriculteurs. Les villages de Souk ElHed et Arbaoua sont choisis (ainsi que deux autres villages non présentés ici). À Souk El-Hed, lorsque la DPA propose le projet au représentant du village qui siège au conseil communal, elle indique qu'il faut une association partenaire. Une association est alors créée spécifiquement pour que le village de Souk El-Hed bénéficie du projet. Cette association comporte sept adhérents, dont cinq de niveau scolaire correspondant à l'école primaire, un membre d'un niveau scolaire correspondant au collège et un membre analphabète. Aucun d'entre eux n'a d'expérience dans une association ou une coopérative, et tous vivent exclusivement de l'agriculture.

L'association de Souk El-Hed et la coopérative d'Arbaoua reçoivent une copie du document global de projet rédigé par la DPA, mais ne reçoivent pas les cahiers des charges décrivant les tâches des entrepreneurs. La DPA lance un appel d'offres pour choisir les entrepreneurs; cependant ni la coopérative d'Arbaoua ni l'association de Souk El-Hed ne sont invitées à l'ouverture des plis. Un responsable du projet au niveau de la DPA a justifié cette absence en arguant que la présence des organisations à cette ouverture n'avait que peu d'intérêt, d'une part, parce que les cahiers des charges sont rédigés en français (langue que les responsables de l'association de Souk El-Hed comprennent peu), et, d'autre part, parce que l'ouverture des plis suit une procédure très normée dans laquelle les agriculteurs n'ont pas de rôle à jouer.

\section{Mise en œuvre}

Dans le village d'Arbaoua, les plantations ont été effectuées en deux tranches, une première de 35 ha pour quinze bénéficiaires en 2010, puis une deuxième de 25 ha pour vingt bénéficiaires en 2011. Deux entrepreneurs ont été sélectionnés pour réaliser la première tranche, et l'un d'entre eux a continué pour la réalisation de la deuxième tranche. En ce qui concerne les autres composantes du projet, 100 ha d'oliviers anciens sont taillés. Du matériel pour la culture des oliviers (vibreurs, pioches, etc.) est distribué. Des traitements sont effectués sur les plantations existantes. Quelques visites et formations sont aussi organisées. En novembre 2011, un pressoir est livré. Cependant, la quantité d'huile d'olive qu'il est capable de produire quotidiennement est inférieure à celle prévue initialement. Le local où est installé le pressoir est construit sur un terrain loué à la coopérative, représentée par son secrétaire, pour dix ans.

Les membres de la coopérative d'Arbaoua ne sont satisfaits ni du travail de l'un des deux entrepreneurs, ni des équipements fournis par l'administration. Ils considèrent que l'entrepreneur n'a pas creusé de trous suffisamment profonds pour planter les plants d'olivier (environ 500 pieds sur des zones en pente, qui avaient été plantés dans des trous creusés à la main, ont toutefois été replantés après inspection de la DPA). La DPA reconnait la médiocre qualité des plantations 
effectuées et, lors de la deuxième tranche, fait appel à un bureau d'étude pour faire le suivi de la qualité de la plantation. Les membres de la coopérative estiment que les entrepreneurs n'ont pas creusé de cuvettes pour l'irrigation et qu'ils n'ont pas assuré le gardiennage. Les agriculteurs se plaignent aussi que les machines pour gauler sont adaptées à de jeunes plants, alors qu'ils possèdent en grande majorité de vieux arbres. Ils considèrent ainsi que la gouvernance du projet aurait pu être améliorée : ils auraient souhaité être présents lors de l'ouverture des plis, notamment pour évaluer les compétences des entrepreneurs. En particulier, ils auraient souhaité empêcher que l'entrepreneur qui n'avait pas répondu à leurs attentes lors de la première tranche soit sélectionné pour la deuxième. Les membres de la coopérative d'Arbaoua auraient aussi souhaité participer au choix des matériels agricoles distribués.

Les trois responsables de la coopérative d'Arbaoua choisissent cependant de ne pas entrer en conflit avec l'administration même lorsqu' un de ses responsables obtient le cahier des charges des entrepreneurs sur Internet et qu'il constate que l'un des entrepreneurs ne le respecte pas du tout. Les responsables de la coopérative choisissent de ne pas mettre en avant ce non-respect du cahier des charges dans leurs relations avec l'administration car, d'une part, ils souhaitent conserver de bonnes relations avec la DPA pour pouvoir obtenir des financements futurs, et, d'autre part, parce qu'ils considèrent que s'ils se mobilisent pour que l'entrepreneur replante des plants mal fertilisés et mal irrigués, ce dernier devra le faire avec de jeunes plants, ce qui retardera de deux années supplémentaires la mise en valeur des terres.

Les composantes du projet sont dans l'ensemble identiques à Souk El-Hed. En 2010, un entrepreneur a planté 50 ha (en zone pluviale) pour 38 bénéficiaires. Selon les agriculteurs interrogés, l'entrepreneur a travaillé peu et mal. Il n'a pas creusé de trous suffisamment profonds et n'a pas utilisé d'engrais. Les agriculteurs se plaignent que, durant l'été 2011, l'entrepreneur n'a irrigué qu'une fois, et une faible surface seulement, et qu'il n'a irrigué que deux fois pendant l'été 2012. De plus, selon eux, l'entrepreneur n'a apporté des engrais qu'aux oliviers situés sur les sols plats et n'a irrigué que ces derniers. Il a délaissé les plants situés sur les parties en pente, où le travail est plus difficile. Selon les agriculteurs, la seule contribution réelle de l'entrepreneur est l'embauche d'une personne du village pour assurer le gardiennage. Par ailleurs, le don de matériel pour le traitement et la récolte a donné lieu à un conflit au sein de l'association. Les membres de l'association ne se sont pas mis d'accord sur les règles d'utilisation du matériel, qui a été réparti entre certains membres et reste remisé chez eux. Le village n’a pas bénéficié d'un pressoir car, en 2012, le projet oléicole a évolué et prévoit désormais d'installer un pressoir de plus grande taille, mis en commun pour l'ensemble des villages faisant partie du projet. 
Les membres de l'association de Souk El-Hed se sont rendus par deux fois à la DPA pour se plaindre du travail de l'entrepreneur, mais ils estiment ne pas avoir obtenu de résultats. Cet échec est venu après celui de la tentative de faire réparer les parties des canaux d'irrigation bétonnés qui s'étaient rompues peu de temps avant le début du projet oléicole. Ces deux échecs ont conforté de nombreux villageois de Souk El-Hed dans leur opinion qu'ils n'ont pas la capacité d'influencer la mise en œuvre des projets de développement. Un agriculteur rencontré sur les terrains plantés dans le cadre du projet oléicole avait ainsi passé plusieurs jours à creuser des cuvettes autour des plants d'oliviers présents sur ses parcelles, car il avait constaté que l'entrepreneur ne venait pas pour le faire. Il prenait acte de cette absence, avec un certain fatalisme.

De son côté, l'entrepreneur interrogé se plaint d'un accès difficile aux oliviers. En effet, les agriculteurs ont semé sur les parcelles, en particulier près des plants d'olivier, bien que la DPA ait fait signer aux présidents de la coopérative d'Arbaoua et de l'association de Souk El-Hed un document stipulant que les agriculteurs ne doivent pas semer dans un cercle de $1,5 \mathrm{~m}$ de diamètre autour de chaque plant. De ce fait, les entrepreneurs ont attendu chaque année la fin de la moisson pour irriguer les plants. Les agents de la DPA reconnaissent que leurs visites de contrôle sur le terrain n'ont pas été aussi fréquentes et détaillées que nécessaires. Ils arguent cependant qu'ils n'ont pas de moyens représentatifs (pas de voitures adéquates pour se rendre dans des zones accidentées, pas de per diem spécifique, etc.) pour mener à bien leur mission.

\section{Conception d'autres projets de développement}

À Souk El-Hed, les agriculteurs ont intégré deux projets correspondant à la plantation d'environ 60 ha d'oliviers et 60 ha d'amandiers en 2013. Cependant, ces deux projets ont été conçus à l'initiative de la DPA et les agriculteurs n'en ont pas élaboré d'autres eux-mêmes. En revanche, dans le village d'Arbaoua, en parallèle à la mise en œuvre du projet oléicole, les trois responsables de la coopérative ont conçu de nombreux autres projets. En 2010, la coopérative obtient des ruches dans le cadre d'un projet financé par l'INDH. L'administration de l'INDH leur indique que, pour obtenir le projet, la coopérative doit s'ouvrir à des femmes. La coopérative intègre ainsi six femmes en 2010. Par ailleurs, en 2012, la coopérative coordonne un projet géré par une université marocaine et une entreprise para-étatique en charge de la commercialisation des produits agricoles. Ce projet permet à la coopérative de réaliser des études pour l'export de son huile d'olive en Europe.

À l'automne 2012, la coopérative d'Arbaoua avait d'autres projets en cours de préparation avec la DPA. Premièrement, les agriculteurs de la coopérative ont eu l'idée, suite au projet oléicole, de proposer un projet pour le caroubier, conçu selon la même logique. Les trois responsables ont fait une demande écrite à la 
DPA, accompagnée d'une fiche technique indiquant les surfaces disponibles et le nombre d'agriculteurs intéressés. Le projet prévoit la plantation de plants de caroubier, la fourniture d'une machine de concassage des graines de caroubier - qui serait gérée en commun avec trois autres coopératives de la région - et une aide à la commercialisation. Malgré le souhait de la coopérative d'Arbaoua d'avoir la machine installée dans son village, la DPA a choisi de l'installer dans une autre coopérative, arguant qu'Arbaoua avait déjà beaucoup bénéficié du plan Maroc vert. Deuxièmement, la coopérative a déposé un projet de collecte de lait dans le cadre du Pilier II du plan Maroc vert. Le projet a été accepté et la coopérative doit bénéficier de l'équipement d'un local pour la réception du lait, d'un bac de 1000 litres, et de bidons en aluminium. La DPA a organisé la création d'une union de cinq jeunes coopératives de collecte de lait dans la province pour qu'elles puissent coordonner les négociations avec des industriels à même d'acheter le lait qu'elles collectent.

Troisièmement, la coopérative a déposé un projet de plantation de 150 ha d'amandiers. Enfin, l'association de développement rural d'Arbaoua a reçu une machine de tissage financée par l'INDH.

Les trois responsables de la coopérative d'Arbaoua qui ont géré le projet oléicole et les projets suivants travaillent en étroite collaboration, chacun ayant un rôle spécifique. Le président n'a que peu de terres; il dédie une part importante de son temps à la gestion de projets de développement et s'occupe en particulier des relations entre la coopérative et les institutions extérieures. Le secrétaire fait partie d'une famille relativement riche par rapport aux autres familles du village (elle possède de nombreuses terres). Il s'occupe des activités agricoles au sein de la coopérative et il a bénéficié de 22 des 40 ha d'oliviers plantés dans le cadre du projet oléicole. Le trésorier est un militaire à la retraite et s'occupe de la comptabilité. Par ailleurs, ces trois personnes ne s'étaient jamais portées candidates aux élections locales.

Les responsables de la coopérative et de l'association qui ont été interrogés n'ont pas mentionné l'implication des membres de base, que ce soit dans la prise de décision ou dans le suivi des activités du projet. De même, les bénéficiaires de l'association de Souk El-Hed qui ont été interrogés ont mentionné une forte méconnaissance du projet oléicole. Dans la coopérative d'Arbaoua comme dans l'association de Souk El-Hed, les décisions concernant le projet oléicole ont été prises au sein d'un petit groupe de personnes, les autres membres restant dans une position de bénéficiaires.

\section{Apprentissage des agriculteurs}

À Arbaoua, le projet d'amélioration de l'activité oléicole, bien que coordonné par l'administration, a permis que les responsables comprennent mieux les procédures du plan Maroc vert. Ce projet oléicole n'était pas le premier projet 
géré par la coopérative. Il est néanmoins important car il s'agit du premier projet de la coopérative d'Arbaoua avec la DPA et qu'il a permis un changement d'échelle dans le financement et le réseau d'acteurs impliqués dans la conception et la mise en œuvre des projets, par rapport à ceux financés par l'INDH. Les trois responsables de la coopérative d'Arbaoua ont appris à comparer les différents types de financement, notamment entre le plan Maroc vert et l'INDH. Les dirigeants de la coopérative ont préféré le plan Maroc vert car les montants alloués sont bien supérieurs et les agriculteurs ne sont pas obligés de contribuer financièrement à la mise en place des projets.

Les responsables de la coopérative d'Arbaoua estiment que les financements du plan Maroc vert et de l'INDH représentent une véritable opportunité, mais ils estiment n'avoir qu'une maîtrise incomplète de leur relation avec les autres acteurs impliqués dans ces projets. Ils vivent comme un échec le fait que la DPA n'ait pas choisi leur village pour l'installation de la machine de concassage des graines de caroubier. De plus, ils estiment n'avoir que peu de contrôle sur les entrepreneurs. Un de ces responsables considère ainsi que « le problème des projets du plan Maroc vert, c'est l'entrepreneur ». Ces dirigeants n'adoptent pas pour autant une logique de rupture avec l'administration et les entrepreneurs : malgré les limites décrites et afin de continuer à décrocher des projets, il faut " de la diplomatie », comme le dit le trésorier de la coopérative.

Au-delà de la formalisation de la procédure d'élaboration de projets qui rend plus visible, pour les agriculteurs, les modalités d'accès à un financement public, l'important est la disponibilité des fonds publics. C'est bien le cas avec le plan Maroc vert et l'INDH, et ce, de façon bien plus importante que dans les années 1990 ou le début des années 2000. Les responsables de la coopérative d'Arbaoua sont conscients des opportunités actuelles. Ils estiment ainsi que "l'argent est là, il suffit de faire des projets ».

Le contraste est fort avec Souk El-Hed, où les responsables interrogés ont appris de l'expérience du projet oléicole, mais ont développé de façon bien moindre leur agencéité. Les agriculteurs de Souk El-Hed se sentent encore largement impuissants face à des acteurs (DPA, entrepreneur) dont ils ne comprennent pas le mode de fonctionnement. Ils expriment un certain fatalisme sur le fait qu'ils ne réussissent pas à influencer le makhzen, c'est-à-dire l'administration marocaine ${ }^{3}$, qui garde les traits d'un acteur lointain et peu accessible. Malgré ce constat largement négatif, le président de l'association de Souk El-Hed estime : « On a beaucoup perdu, mais on a aussi beaucoup appris. » Il donne à l'association l'objectif d'obtenir le cahier des charges de l'entrepreneur

3. Le makhzen désigne littéralement l'« entrepôt ». " Jusqu'au protectorat, il faisait référence à la maison royale puis à l'appareil d'État de l'empire chérifien. » (Hibou, 2006). Aujourd'hui, le makhzen représente à la fois l'État (dont les limites ne sont jamais explicitées) et un mode de gouvernement centralisé. 
dès le début de la mise en œuvre des prochains projets, et d'effectuer le contrôle de chacune des actions de l'entrepreneur pour vérifier sa conformité avec les normes. Pour résumer : «Il faudra qu'on sache tout dans le détail. »

Ces différences d'apprentissage conduisent aussi à des différenciations en ce qui concerne l'impact des projets. La différenciation la plus évidente est celle entre Arbaoua et Souk El-Hed en termes de dynamisme économique lié aux projets. Une autre différenciation existe au sein même du village d'Arbaoua. Les villageois non membres de la coopérative paient, pour pouvoir utiliser le pressoir de la coopérative, un tarif deux fois plus élevé que celui des membres. Quant au projet de coopérative laitière, il est fondé sur l'objectif explicite de collecter une grande partie du lait produit dans le village, tandis que seuls les vingt membres de la coopérative toucheront les bénéfices que cette entreprise pourrait générer. À la question d'une possible augmentation du nombre d'agriculteurs bénéficiant des financements publics dans le cadre de la coopérative d'Arbaoua, les responsables de cette dernière répondent qu'ils refusent d'augmenter le nombre d'adhérents, arguant qu' ils ont peur de ne plus arriver à gérer les conflits. Ils mettent cependant en avant l'aide qu'ils apportent à la conception de deux autres coopératives dans des villages de la région et l'accompagnement d'un projet de création d'une autre coopérative laitière à Arbaoua. Certains agriculteurs d'Arbaoua, non bénéficiaires des différents projets de la coopérative, voient cependant cette dernière comme une entité fermée sur elle-même et estiment que ses membres ne cherchent pas à aider les autres agriculteurs du village à créer des structures comparables.

\section{Point de vue de l'administration}

Suite à une première vague de projets dans le cadre du plan Maroc vert, l'administration du ministère de l'Agriculture a cherché à mieux cadrer la participation des agriculteurs. Elle a ainsi modifié le guide de procédure du Pilier II en 2013 pour y inclure la nécessité de la demande de la conception des projets à l'initiative d'une organisation professionnelle ou d'un organisme équivalent (Agence de développement agricole, 2015). De plus, une circulaire du ministère de l'Agriculture et des Pêches maritimes de février 2012 stipule que le président de l'organisation professionnelle porteuse du projet doit participer à la préparation des cahiers des charges et doit être présent à l'ouverture des plis et à la réception des travaux. Cependant, cette prise de responsabilité, réelle mais limitée, ne va pas jusqu'à l'ambition d'une définition concertée d'un développement rural sur le long terme, qui permettrait notamment de dépasser l'approche filière $e^{4}$ des projets du plan Maroc vert.

4. Dans le secteur agricole, l'approche filière s'intéresse aux interactions entre les acteurs économiques intervenant dans la production, possiblement la transformation, et la commercialisation d'un produit agricole. 
Par ailleurs, dans la province où se trouve le village d'Arbaoua, quelques autres collectifs d'agriculteurs ont eux aussi appris à concevoir des projets, notamment de coopératives de collecte de lait, et à demander un financement à la DPA pour ces projets. Il y a ainsi, dans cette province, une distinction claire entre les projets de la première vague du plan Maroc vert, initiés entre 2007 et 2009, et les projets suivants, dont plusieurs ont été à l'initiative des agriculteurs. Cependant, l'administration ne s'est pas donné les moyens d'accompagner la conception de projets auprès de groupes qui n'en auraient pas initialement les capacités. Certains ingénieurs de la DPA comptent sur un effet " tâche d'huile ", par lequel d'autres collectifs se créent pour concevoir des projets lorsqu' ils voient les succès de la première vague. Certains ingénieurs de la DPA sont conscients du fait que, dans la situation actuelle, les agriculteurs n'ont pas tous les mêmes capacités à concevoir des projets, mais ils remarquent également que la DPA ne dispose ni d'un budget ni du temps officiellement aménagé pour accompagner des collectifs d'agriculteurs ayant de faibles capacités dans la conception d'un projet de développement.

\section{DISCUSSION}

\section{Des agencéités croissantes, mais qui restent liées à une logique de captation de ressources}

Les trois responsables d'Arbaoua ont appris à mieux comprendre les institutions publiques et, comme l'inclusion des femmes dans la structure le montre, les actions à mener pour obtenir des financements. Ils ont montré des capacités de prise d'initiative pour intégrer les nouvelles procédures de financement de projets de l'administration. Ces capacités vont ainsi au-delà de l'influence dans le cadre de relations informelles, telle qu'identifiée par Bouzidi (2012). Comme le mentionnent Corbridge et al. (2005), le caractère formalisé et pérenne des nouvelles procédures de financement de projets a permis de rendre plus compréhensible et un peu plus prévisible l'action de l'État. Les responsables d'Arbaoua sont cependant bien moins entrés dans une négociation détaillée des composantes des projets que les agriculteurs participant aux projets étudiés par Thomas (2013).

Malgré ces niveaux d'agencéité accrus, il n'y a eu, ni du côté de l'administration ni de celui des agriculteurs, de tentatives pour construire une relation de partenariat durant la conception et la mise en œuvre du projet oléicole. En particulier, le projet n'a pas été conçu dans le cadre d'une vision à long terme du développement local. Le choix d'investir dans la production oléicole a répondu à des objectifs d'augmentation de la production au niveau national, non à un diagnostic des difficultés et opportunités de développement dans les deux villages où le projet a été réalisé. 
Bono (2012) et Bergh (2010) ont souligné que les relations entre agriculteurs et administration restent fondées sur une logique de distribution et de captation de ressources plutôt qu'une logique de co-construction d'un projet commun. Cette logique est aussi à l'œuvre dans le projet du plan Maroc vert étudié ici. Elle est liée à un manque général de procédures pour que l'administration et les entrepreneurs rendent des comptes aux agriculteurs (Bergh, 2012). Mais aussi, face à un État «bailleur de fonds » (Bono, 2010), les responsables de la coopérative d'Arbaoua préfèrent la logique de l'accommodement plutôt que celle de la contestation. Dans cette logique de captation de ressources, les agriculteurs d'Arbaoua choisissent de n'entrer en conflit ni avec la DPA ni avec l'entrepreneur. Comme dans le cas d'un projet majeur d'infrastructure dans le Nord du Maroc (Planel, 2011), les habitants des zones rurales étudiées ici préfèrent aussi agir à l'intérieur du cadre posé par l'administration, plutôt qu'initier une confrontation.

\section{Une différenciation des agencéités}

Les nouvelles formes d'agencéité des agriculteurs d'Arbaoua sont fondées sur différentes ressources. Il s'agit d'abord des compétences des responsables de la coopérative, qui ont compris le fonctionnement et les attentes de l'administration. Ces trois responsables ont développé leurs compétences et leur agencéité essentiellement par le biais des activités menées au sein de la coopérative et de l'association de développement rural. Les réseaux dans lesquels ils sont insérés constituent une autre ressource. Le président de la coopérative dédie une part importante de son temps à des activités de courtier de développement (Bierschenk et al., 2000). Ces réseaux sont avant tout tissés avec des personnels de l'administration du ministère de l'Agriculture et avec ceux du ministère de l'Intérieur, en charge de la gestion de l'INDH. La maîtrise de relations de proximité entre notables et personnel de l'administration (telles que décrites dans El Iraki, 2002) n'est plus nécessaire à l'obtention de financements. De plus, les dirigeants de la coopérative d'Arbaoua ont tissé de nombreux liens au niveau provincial, national et même international, grâce au projet d'exportation d'huile d'olive. Par contraste, les responsables de l'association de Souk El-Hed n'ont de contact à aucun de ces niveaux. Enfin, les responsables de la coopérative d'Arbaoua ont acquis une forte expérience de l'action collective. Ils font partie d'une association depuis plus de quinze ans et ils ont eu à gérer des conflits par le passé. Ils ont analysé ces conflits et en ont tiré des leçons. En comparaison, les membres de l'association de Souk El-Hed n'ont pas su gérer le premier conflit apparu au sein de l'association, autour de la distribution du matériel.

Au Maroc, l'instauration de dispositifs formalisés et pérennes de conception de projets de développement local, avec une composante officiellement participative, conduit ainsi à une nouvelle structure d'opportunité où l'agencéité des 
agriculteurs nécessite des ressources différentes de celles mobilisées par le passé. Notre étude montre la forte différence qui peut exister entre différents groupes d'agriculteurs quant à la maitrise de ces nouvelles ressources.

Par rapport à l'étude de Mansuri et Rao (2012), notre étude montre que cette agencéité des agriculteurs dépend bien sûr de ressources spécifiques aux individus (niveau d'instruction, etc.), mais aussi que cette agencéité est dynamique. Elle évolue avec la participation à des projets du fait d'une meilleure compréhension des procédures administratives, du renforcement des réseaux avecl'administration et de l'expérience de l'action collective. Ceci rejoint l'analyse des courtiers de développement faite par Bierschenk et al. (2000), qui montre que ces courtiers apprennent et construisent leur agencéité au fur et à mesure de leur participation à des projets de développement.

Faysse et al. (2015) montrent que la différenciation des compétences entre responsables et membres de base est un fait au sein d'associations et coopératives au Maroc, d'autant plus lorsque les personnes en charge de la gestion du groupe restent longtemps à leur poste et n'impliquent pas l'ensemble des membres dans les prises de décision. Cette différenciation est aussi à l'œuvre dans le projet de développement étudié ici. Dans la nouvelle structure d'opportunité des projets de développement rural et agricole au Maroc, la différentiation des compétences et de l'agencéité peut avoir lieu ainsi à deux niveaux : entre différents groupes (tels que la coopérative d'Arbaoua et l'association de Souk El-Hed), mais aussi au sein même de chaque groupe.

\section{CONCLUSION}

Le plan Maroc vert a instauré des procédures novatrices de conception de projets de développement, qui s'affichent comme participatives. Dans les deux cas étudiés, les résultats sont ambivalents. D'un côté, les collectifs d'agriculteurs des deux villages n'ont disposé que d'une agencéité très limitée pour influencer la conception et la mise en œuvre du projet oléicole. De l'autre, les responsables d'un des groupes ont fortement développé des compétences de conception de projets à même d'être financés dans le cadre du plan Maroc vert et de l'INDH, et ont ainsi obtenu différents financements pour réaliser leurs propres actions.

Cependant, cette amélioration de l'agencéité de certains habitants des zones rurales n'est pas le résultat d'une posture volontariste des institutions publiques, qui chercheraient plutôt à intégrer les agriculteurs dans un processus de construction conjointe des projets de développement. Elle a plutôt été permise par le fait que les dispositifs de financement sont devenus pérennes et plus compréhensibles par les agriculteurs. Ceci justifie d'ailleurs, ex post, l'intérêt d'avoir mené dans la présente étude une approche centrée sur l'agencéité des agriculteurs, car une analyse qui aurait été centrée sur le degré de participation 
des agriculteurs à la procédure de conception du projet oléicole se serait limitée au constat d'une faible participation.

Ainsi, l'agencéité accrue de certains groupes d'agriculteurs apparait comme un effet largement involontaire de politiques centrées sur l'amélioration de la production agricole (plan Maroc vert) ou sur l'amélioration des revenus (programme d'activité génératrice de revenus de l'INDH). Ceci conduit à deux risques. D'une part, il se peut que cette agencéité renforcée continue, dans le futur, de s'exprimer dans le cadre d'une relation fondée sur une logique de distribution de ressources, et que les relations entre État et agriculteurs n'évoluent pas vers un cadre de partenariat et une coresponsabilité dans la prise de décisions. D’autre part, les ressources dont disposent les habitants des zones rurales pour agir dans cette nouvelle structure d'opportunité sont très hétérogènes. En l'absence d'un accompagnement spécifique de l'État, une différenciation croissante peut se développer entre une minorité capable d'obtenir le financement de nombreux projets et une majorité exclue des nouveaux dispositifs de développement. Il est important que les dispositifs de développement organisés dans le cadre du plan Maroc vert et de l'INDH renforcent les capacités des habitants en zone rurale, en particulier leur agencéité, et ce, plus spécialement pour les populations qui ont actuellement de moindres capacités d'interaction avec les institutions de développement.

\section{BIBLIOGRAPHIE}

Agence de développement agricole, 2015, Procédure de mise en cuvre des projets Pilier II du plan Maroc vert. http://www. ada.gov.ma/FichiersActualite/Fichier_ DocTelecharger20150612095831.pdf (novembre 2015).

Alsop R., Bertelsen M. F., Holland, J., 2006, Empowerment in Practice: From Analysis to Implementation, Washington DC, Banque mondiale.

Bergh S., 2010, « Assessing the Scope for Partnerships Between Local Governments and Community-Based Organizations: Findings from Rural Morocco », International Journal of Public Administration, vol. 33, $\mathrm{n}^{\circ}$ 12-13, pp. 740-751, http://www.tandfonline.com/ doi/full/10.1080/01900692.2010.513358 (octobre 2015).
Bergh S., 2012, "Inclusive" Neoliberalism, Local Governance Reforms and the Redeployment of State Power: The Case of the National Initiative for Human Development (INDH) in Morocco ", Mediterranean Politics, vol. 17, $\mathrm{n}^{\circ} 3$, pp. 410-426, http://www.tandfonline.com/doi/full/ 10.1080/13629395.2012.725304 (octobre 2015).

Bierschenk T., Chauveau J.-P., Olivier de Sardan J.-P., 2000, Courtiers en développement. Les villages africains en quête de projets, Paris, Karthala.

Bono I., 2010, "L'activisme associatif comme marché du travail. Normalisation sociale et politique par les "activités génératrices de revenu" à El Hajeb », Politique africaine, $n^{\circ} 120$, pp. 25-44. 
Bono I., 2012, " 'L'INDH n'achète que des vaches!" Mobilisation de ressources et notabilité à l'épreuve du développement au Maroc », in Abu-Sada C., Challand B. (dir.), Le développement, une affaire d'ONG ? Associations, États et bailleurs dans le monde arabe, Paris, Karthala.

Bouzidi Z., 2012, Dénouer les fils de la coordination à travers l'appréhension des grammaires locales. Analyse des pratiques de coordination pour la gestion des ressources productives dans le périmètre du Gharb au Maroc, Thèse de doctorat, Paris, Université Paris 10.

Chauveau J.-P., 1994, «Participation paysanne et populisme bureaucratique. Essai d'histoire et de sociologie de la culture du développement », in Jacob J.-P., Lavigne-Delville P. (dir.), Les associations paysannes en Afrique. Organisations et dynamiques, Paris, Karthala.

Corbridge S., Williams G., Srivastava M. et al. (dir.), 2005, Seeing the State: Governance and Governmentality in India, Cambridge, Cambridge University Press.

De Herdt T., Bastiaensen J., 2009, «L'Agencéité relationnelle ", Revue Tiers Monde, n 198 , pp. 317-333, http://www.cairn.info/ revue-tiers-monde-2009-2-page-317.htm (octobre 2015).

De Miras C., 2007, «Initiative nationale pour le développement humain et économie solidaire au Maroc pour un accès élargi à l'eau et à l'assainissement », Revue Tiers Monde, n 190, pp. 357-377, http://www.cairn.info/ revue-tiers-monde-2007-2-page-357.htm (octobre 2015).

Direction des aménagements forestiers, 2002, " Stratégie de développement des zones bour à travers la mise en œuvre de la loi 33-94 relative aux périmètres de mise en valeur en bour ", Hommes, terres et eaux, $n^{\circ} 122$, pp. 7-11, http://www.anafide.org/doc/HTE\% 20122/122-2.pdf (octobre 2015).

El Iraki A., 2002, Des notables du Makhzen à l'épreuve de la "gouvernance ", Paris, L'Harmattan.
El Jihad, M.D., 2010, " Les difficultés de gestion des ressources "naturelles" et de développement rural dans un milieu anthropisé : l'expérience du projet Oued Srou (Maroc central) ", Norois, n²16, pp. 25-45, http://norois.revues.org/3320 (octobre 2015).

El Mkadmi S., 2011, Analyse de la mise en place et de l'accompagnement technique des projets du deuxième pilier du plan Maroc vert : Cas du projet olivier dans la région de Meknès, Mémoire de fin d'études, Meknès, École nationale d'agriculture de Meknès.

Faysse N., 2015, "The Rationale of the Green Morocco Plan: Missing Links between Goals and Implementation », Journal of North African Studies, vol. 20, $\mathrm{n}^{\circ} 4$, pp. 622-634, http://www.tandfonline.com/ doi/full/10.1080/13629387.2015.1053112 (octobre 2015).

Faysse N., Errahj M., Kuper M. et al., 2010, "Learning to Voice? The Evolving Roles of Family Farmers in the Coordination of Large-Scale Irrigation Schemes in Morocco ", Water Alternatives, vol. 3, n 1, pp. 48-67, http://www.water-alternatives.org/index.php/ alldoc/articles/vol3/v3issue1/70-a3-1-3/file (octobre 2015).

Faysse N., Bouzekraoui M., Errahj M., 2015, "Participation et apprentissages dans des groupes restreints. Cas de coopératives féminines au Maroc », Revue d'anthropologie des connaissances, vol. 9, $\mathrm{n}^{\circ} 3$, https://www.cairn.info/revue-anthropologiedes-connaissances-2015-3-page-351.htm (octobre 2015).

Giddens A., 1984, The Constitution of Society: Outline of the Theory of Structuration, Berkeley, University of California Press.

Hibou B., 2006, «Maroc : d'un conservatisme à l'autre », in Bayart J.-F. et al., Legs colonial et gouvernance contemporaine, Vol. 2, Paris, Fasopo, http://www.fasopo.org/sites/ default/files/legscolonial2_bh_1206.pdf (octobre 2015). 
Kadiri Z., 2012, Évolution du contenu et du contour de l'action publique au Maroc dans le domaine de l'eau d'irrigation : Entre logique paysanne et logique technique, Thèse de doctorat, Aixen-Provence, Université d'Aix-Marseille.

Lazarev G., 2012, Les politiques agraires au Maroc, 1956-2006. Un témoignage engagé, Rabat, Économie critique, http://www.amse.ma/doc/Politiques\% 20agraires\%20Lazarev.pdf (octobre 2015).

Le Meur P.Y., 2000, « Courtage en développement et champ du pouvoir local au Sud-Bénin ", in Bierschenk T. et al. (dir.), Courtiers en développement. Les Villages africains en quête de projets, Paris, Karthala.

Leveau R., 1976, Le fellah marocain défenseur du trône, Paris, Presses de la Fondation nationale des sciences politiques.

Long N., 2002, Development Sociology: Actor Perspectives, Londres, Routledge.

Mansuri G., Rao V., 2012, Localizing Development: Does Participation Work? Washington DC, Banque mondiale.

Montanari B., Bergh S., 2014, "The Challenges of "Participatory" Development in a Semi-Authoritarian Context: the Case of an Essential Oil Distillation Project in the High Atlas Mountains of Morocco", The Journal of North African Studies, vol. 19, n 5 , pp. 828-851, http://www.tandfonline.com/ doi/full/10.1080/13629387.2013.878247 (octobre 2015).

Mosse D., 2005, Cultivating Development: an Ethnography of Aid Policy and Practice, Londres, Pluto Press.

Olivier de Sardan J.-P., 2008, La rigueur du qualitatif. Les contraintes empiriques de l'interprétation socioanthropologique, Paris, L'Harmattan.

Planel S., 2011, "Mobilisations et immobilisme dans l'arrière-pays de Tanger-Med. Effet des contradictions de la réforme de l'État ", Revue Tiers Monde, Hors série, pp. 189-206, http://www.cairn.info/revue-tiers-monde2011-5-page-189.htm (octobre 2015).

Préfol P., 1986, Prodiges de l'irrigation au Maroc, le développement exemplaire du Tadla (19361985), Paris, Nouvelles Éditions latines.

Robins S., Cornwall A., von Lieres B., 2008, "Rethinking "Citizenship" in the Postcolony ", Third World Quarterly, vol. 29, n 6, pp. 10691086, http://www.tandfonline.com/doi/full/10. 1080/01436590802201048 (octobre 2015).

Thomas L., 2013, Rôle de l'agriculture familiale dans la conception des projets plan Maroc vert Pilier II. Exemple de la filière pomme, Mémoire de master, Paris, AgroParisTech. 